\title{
Traveling-Wave Electrokinetic Micropumps: Velocity, Electrical Current, and Impedance Measurements
}

\author{
P. García-Sánchez, ${ }^{* \dagger}$ A. Ramos, ${ }^{\dagger}$ N. G. Green, ${ }^{*}$ and H. Morgan ${ }^{\ddagger}$ \\ Departamento de Electrónica y Electromagnetismo, Facultad de Física, Universidad de Sevilla, 41012 \\ Sevilla, Spain, and School of Electronics and Computer Science, University of Southampton, \\ SO17 1BJ Southampton, U.K.
}

\author{
Received February 7, 2008. Revised Manuscript Received May 6, 2008
}

\begin{abstract}
An array of microelectrodes covered in an electrolyte and energized by a traveling-wave potential produces net movement of the fluid. Arrays of platinum microelectrodes of two different characteristic sizes have been studied. For both sizes of arrays, at low voltages $\left(<2 \mathrm{~V}_{\mathrm{pp}}\right)$ the electrolyte flow is in qualitative agreement with the linear theory of ac electroosmosis. At voltages above a threshold, the direction of fluid flow is reversed. The electrical impedance of the electrode-electrolyte system was measured after the experiments, and changes in the electrical properties of the electrolyte were observed. Measurements of the electrical current during pumping of the electrolyte are also reported. Transient behaviors in both electrical current and fluid velocity were observed. The Faradaic currents probably generate conductivity gradients in the liquid bulk, which in turn give rise to electrical forces. These effects are discussed in relation to the fluid flow observations.
\end{abstract}

\section{Introduction}

Advances in laboratory-on-a-chip technology or micro total analysis systems ( $\mu$ TAS) demands the precise control of fluids in small channels (a process called microfluidics). ${ }^{1}$ A wide variety of techniques have been developed to pump liquids in microsystems. ${ }^{2}$ For example, electrical forces applied directly to the liquid enable pumping of small amounts of liquids without moving parts. Electroosmosis, ${ }^{3}$ electrowetting, ${ }^{4}$ ion-drag pumping ${ }^{5}$ electrohydrodynamic induction pumping, ${ }^{6}$ and ac electroosmosis ${ }^{7}$ are examples of electric-field-driven micropumping. ${ }^{8}$

In this work, we study experimentally the pumping of electrolytes using ac electroosmosis (ACEO) ${ }^{9,10}$ ACEO pumping is based on the use of arrays of microelectrodes driven by a low potential ac signal (less than $10 \mathrm{~V}$ ). The arrays of planar microelectrodes are mounted in a microfluidic channel, and net fluid flow is observed after application of an electric potential. The current theory for ACEO assumes that the liquid is driven at the surface of the electrodes by the lateral force that the electric field exerts on the induced charge in the diffuse part of the electrical double layer. Unidirectional fluid flow is obtained using either arrays of pairs of asymmetric electrodes subjected to a

* Corresponding author. E-mail: pablogarcia@us.es.

${ }^{\dagger}$ Universidad de Sevilla.

* University of Southampton.

(1) Stone, H.; Stroock, A.; Ajdari, A. Annu. Rev. Fluid Mech. 2004, 36, 381411.

(2) Laser, D. J.; Santiago, J. G. J. Micromech. Microeng. 2004, 14, R35-R64.

(3) Pretorius, V.; Hopkins, B.; Schieke, J. J. Chromatogr. 1974, 99, 23-30.

(4) Beni, G.; Tenan, M. J. Appl. Phys. 1995, 52, 6011-6015.

(5) Richter, A.; Sandmaier, H. An Investigation of Micro Structures, Sensors, Actuators, Machines and Robots; In Proceedings of Micro Electro Mechanical Systems; Institute of Electrical and Electronics Engineers: New York, 1990 ; pp 99-104.

(6) Fuhr, G.; Hagedorn, R.; Muller, T.; Benecke, W.; Wagner, B. J. Microelectromech. Syst. 1992, 1, 141-146.

(7) Brown, A. B. D.; Smith, C. G.; Rennie, A. R. Phys. Rev. E 2000, 63, 016305 .

(8) Ramos, A. In Microfluidics Technologies for Miniaturized Analysis Systems; Hardt, S., Schönfeld, F. H., Eds.; Springer: New York, 2008; Electrohydrodynamics and Magnetohydrodynamics Micropumps.

(9) Ramos, A.; Morgan, H.; Green, N.; Castellanos, A. J. Colloid Interface Sci. 1999, 217, 420-422.

(10) Green, N.; Ramos, A.; González, A.; Morgan, H.; Castellanos, A. Phys. Rev. E 2000, 61, 4011-4018.

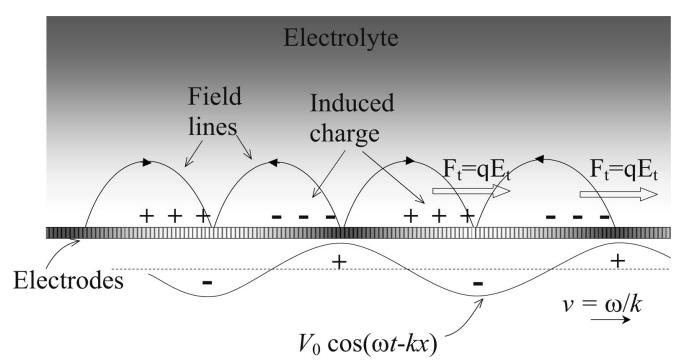

Figure 1. Diagram illustrating the physical mechanism of travelingwave electroosmosis.

single-phase ac signal ${ }^{7,11-13}$ or arrays of equally sized electrodes with a traveling wave signal. ${ }^{14,15}$ Figure 1 shows the basic mechanism for the case of a traveling wave potential. The electric field induces charges in the double layer. The charging of the double layer is analogous to the charging of a capacitor $C$ (the double layer) through a resistor $R$ (the ohmic liquid); therefore, the characteristic charging frequency is $\omega_{\mathrm{c}}=1 / R C$. Because of the finite time in which the double layer is charged, a delay exists between the time of maximum induced charge and the maximum applied signal. The tangential component of the electric field acts on the charge pulling the fluid in the direction of the traveling wave. Theory predicts a net flow in the direction of the traveling wave. The tangential electrical force depends on the frequency of the applied signal. For frequencies much greater than the characteristic charging frequency $\omega_{\mathrm{c}}$, the induced charge is small. However, for frequencies much smaller than $\omega_{\mathrm{c}}$ the induced charge is maximized, but now there is no time delay between the ac signal and the induced charge so no tangential force occurs. A bell-shaped curve is predicted for the velocity versus frequency data with the maximum velocity around $\omega_{\mathrm{c}}$.

(11) Ajdari, A. Phys.Rev. E 2000, 61, R45-R48.

(12) Studer, V.; Pépin, A.; Chen, Y.; Ajdari, A. Analyst 2004, 29, 944-949.

(13) Urbanski, J.; Thorsen, T.; Levitan, J.; Bazant, M. Appl. Phys. Lett. 2006, $89,143508$.

(14) Cahill, B. P.; Heyderman, L. J.; Gobrecht, J.; Stemmer, A. Phys. Rev. E 2004, 70, 036305.

(15) Ramos, A.; Morgan, H.; Green, N. G.; González, A.; Castellanos, A. J. Appl. Phys. 2005, 97, 084906. 


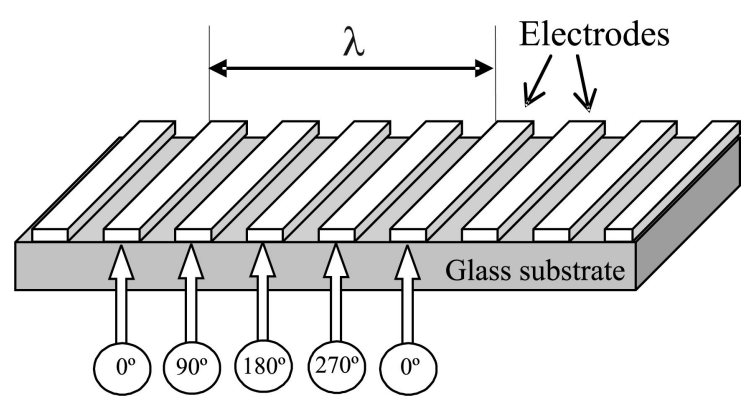

Figure 2. Diagram of the traveling-wave electrode array energized with four phase-shifted voltage sources.

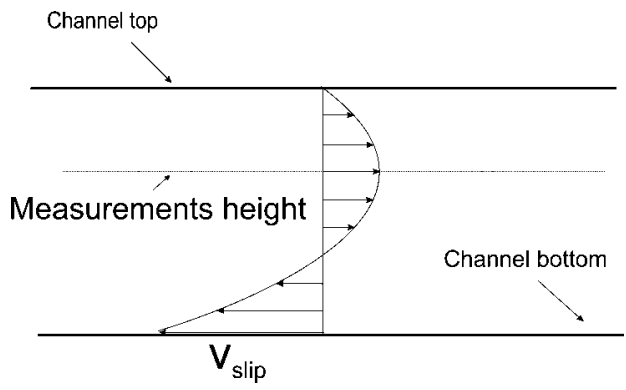

Figure 3. Vertical profile of the fluid velocity in a closed channel. The fluid is driven at the level of the electrodes and recirculates. Velocities are measured at a distance that is $2 / 3$ of the channel height above the electrodes where the velocity has a local maximum.

The predictions of the ACEO theory reasonably explain experimental observations at voltages $\leq 5 \mathrm{~V}_{\mathrm{pp}}$ when using pairs of symmetric electrodes, ${ }^{10,16}$ as well as those at voltages $\leq 2-3$ $\mathrm{V}_{\mathrm{pp}}$ with arrays of electrodes. ${ }^{7,12,14,15,17 \text { Experiments at higher }}$ voltages cannot be explained by the theory; even the direction of the net flow is opposite to prediction. The theory for low voltages is apparently satisfactory for sufficiently low amplitude signals. However, recent experiments with an array of asymmetric pairs of platinum microelectrodes ${ }^{18}$ show pumping in the opposite direction at voltages below $2 \mathrm{~V}_{\mathrm{pp}}$ but only for electrolyte concentrations equal to or greater than $400 \mu \mathrm{M}$. The ACEO theory ${ }^{19,20}$ is at present insufficient to explain all of the experimental observations. ${ }^{12,15}$

In this article, we study the pumping of electrolytes using traveling-wave electrode arrays made of platinum (Figure 2). A sketch of the fluid velocity profile produced over the electrodes is shown in Figure 3. The electrodes are of equal width and gap and energized with a four-phase ac signal. We previously reported experiments where electrolytes $(\mathrm{KCl}$ in water) were pumped with an array of titanium electrodes of $20 \mu \mathrm{m}$ width. ${ }^{21}$ We now present experimental results using platinum electrodes of two different widths (10 and $20 \mu \mathrm{m})$. The different traveling-wave arrays show similar qualitative behavior. At low voltage, the liquid flows in the direction predicted by the theory of ac electroosmosis (i.e., in the direction of the traveling wave). Above a threshold voltage that depends on the applied signal frequency, the fluid flow is reversed. The threshold voltage also depends on

(16) Green, N. G.; Ramos, A.; González, A.; Morgan, H.; Castellanos, A. Phys. Rev. E 2002, 66, 026305-2002.

(17) Mpholo, M.; Smith, C. G.; Brown, A. B. D. Sens. Actuators B 2003, 92, $262-268$.

(18) Gregersen, M. M.; Olesen, L. H.; Brask, A.; Hansen, M. F.; Bruus, H. Phys. Rev. E 2007, 76, 056305.

(19) Olesen, L.; Bruus, H.; Ajdari, A. Phys. Rev. E 2006, 73, 056313.

(20) Ramos, A.; González, A.; García-Sánchez, P.; Castellanos, A. J. Colloid Interface Sci. 2007, 309, 323-331.

(21) García-Sánchez, P.; Ramos, A.; Green, N. G.; Morgan, H. IEEE Trans. Dielectr. Electr. Insul. 2006, 13, 670-677. the array characteristics (i.e., material and size).

We also report electrical impedance measurements of the system before and after the array has been pumping the electrolyte for $5 \mathrm{~min}$. After the traveling wave potential was switched off, the impedance of the array was measured as a function of time. The measurements indicate a sharp decrease in the impedance of the system compared with the values prior to application of the signal. Continued monitoring of the impedance showed that the original values were recovered after around $100 \mathrm{~s}$. The electrical current was measured while the array was pumping the liquid, and transient behaviors in both electrical current and fluid velocity were observed.

This is the first time that electrical current and impedance measurements have been correlated with observations of fluid flow. Changes in the liquid occur at frequencies and voltages typical of ACEO, and this may have important consequences for fluid flow. Current theories for ACEO take into account only forces in the diffuse layer; they do not consider changes in the liquid properties with time and have not provided an explanation for reverse flow. The only mechanism proposed for reversal at higher frequencies considers ionic steric effects plus different charging times for electrodes of different widths. ${ }^{22}$ This scenario is not true for our systems where the electrodes are all of the same width. Changes in liquid properties could enable other EHD effects to drive the flows, including electric forces acting beyond the Debye length (i.e., outside the diffuse layer).

The article is organized as follows: First, experimental details are explained; second, we present results of fluid velocity measurements for the different arrays and make comparisons with previous experiments (using titanium electrodes). Third, the electrical characterization of the system is reported, with simultaneous measurements of velocity and electrical current. Finally, we discuss the experimental results and revisit the possible origins of the electrical forces beyond the Debye length.

\section{Experimental Details}

The microelectrode array used for traveling-wave (TW) pumping experiments is shown in Figure 2. The device consists of a row of 55 coplanar microelectrodes of equal size fabricated on a planar glass wafer using photolithography. Two arrays were studied-array A consisting of $20-\mu \mathrm{m}$-wide electrodes separated by $20 \mu \mathrm{m}$ gaps and array B consisting of $10-\mu \mathrm{m}$-wide electrodes with $10 \mu \mathrm{m}$ gaps. The total length of array A is $2.2 \mathrm{~mm}$, and that of array B is $1.1 \mathrm{~mm}$. Each electrode is driven by an ac voltage $V_{i}(t)=V_{0} \cos \left(\omega t+\phi_{i}\right)$. The difference in phase between consecutive electrodes is $\phi_{i+1}-$ $\phi_{i}=90^{\circ}$, as shown in Figure 2. This produces a traveling-wave potential with a wavelength of $\lambda=160 \mu \mathrm{m}$ for array A and $\lambda=80$ $\mu \mathrm{m}$ for array B. Microscope coverslips were used to fabricate a fluid channel (approximately $3 \mathrm{~cm}$ long) over the microelectrode array with dimensions of $700 \mu \mathrm{m}$ width by $180 \mu \mathrm{m}$ height. A $10^{-4} \mathrm{M}$ solution of $\mathrm{KCl}$ (conductivity is $1.5 \mathrm{mS} / \mathrm{m}$ ) was used as the working electrolyte. The ends of the channel were connected to PTFE tubes and thence to a syringe. The electrolyte was renewed after every measurement. Fluorescent latex particles (500 nm diameter) were suspended in the electrolyte and used as flow tracers. An epifluorescence microscope (Nikon Optiphot-100) was used to observe the flow tracers, recorded using a digital camera and video. Fluid velocity measurements were carried out using particle tracking velocimetry (PTV).

Electrical current measurements were made by energizing the array with a four-output-signal generator. Current was measured from the voltage drop across four identical resistors (of low resistance, $10 \Omega$ ) connected to the device (Figure $4 \mathrm{a}$ ). The voltage drop was measured using a lock-in amplifier, where the in-phase $V_{x}$ and outof-phase $V_{y}$ signals were measured with respect to the driving signal.

(22) Storey, B.; Kilic, M.; Bazant, M. Phys. Rev. E 2008, 77, 036317. 


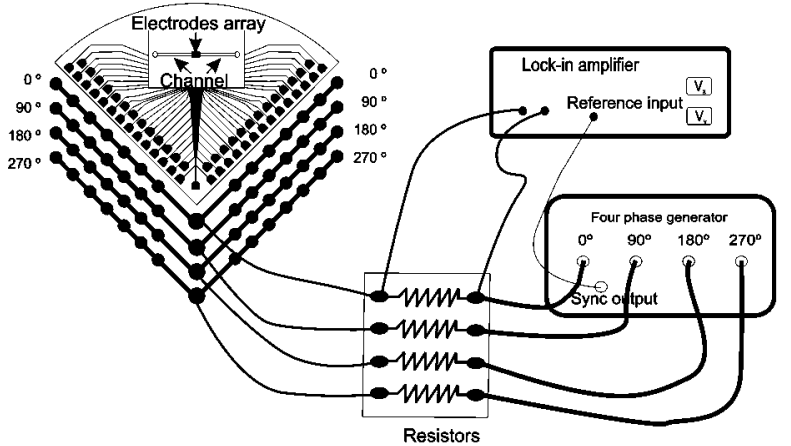

(a) Connections for the electrical current measurement. The voltage drop across a resistor is measured by the Lock-in amplifier.

Figure 4. Device connections and setup for electrical measurements.

The impedance of the array was measured using an impedance analyzer (Agilent 4294A). The impedance of the device was measured before and after application of the traveling-wave signals for pumping. Electrodes corresponding to phases of $0^{\circ}$ and $180^{\circ}$ are connected together to the high output of the impedance analyzer; similarly, electrodes corresponding to phases of $90^{\circ}$ and $270^{\circ}$ are connected together to the low output (Figure 4b). In this way, the impedance is measured between neighboring electrodes and gives a measure of the quasi-equilibrium properties of the liquid before and after the traveling-wave potential is applied.

\section{Velocity Measurements}

When an ac voltage is applied to the electrode arrays, the fluid is driven into flow close to the electrode surface. Because the channel is closed with valves at both ends (input and output), the fluid recirculates, and the tracer particles at the top move in the opposite direction to those above the electrodes (Figure 3). The pump behavior was characterized as a function of voltage and frequency by measuring the fluid velocity at a height of 120 $\mu \mathrm{m}$ above the electrodes, which is $2 / 3$ of the channel height. At this point, the fluid is moving in the opposite direction to that at the electrode surface, and the velocity measurements can be considered to be representative of the global behavior of the pump. Figure 3 shows the theoretical velocity profile obtained as a consequence of a slip boundary condition at the electrodes arrays and a nonslip at the top of the channel. A similar profile was observed experimentally in previous work. ${ }^{21}$ The observed velocity profiles could correspond to fluid flow generated close to the electrodes but not necessarily at the electrode-electrolyte interface. Because the height of the channel is much greater than the electrode width, forces applied at the electrode surface or forces applied within a region the size of the electrode width would generate similar velocity profiles.

Figure 5 shows a 2-D map of the velocity measurements for traveling-wave array A (20- $\mu \mathrm{m}$-wide electrodes). Every measurement was taken using the following protocol: new liquid was flushed through the microchannel, the signal generator was switched on, and videos of the flow tracers were captured during the next $20 \mathrm{~s}$. The measurements were plotted in the voltage versus frequency domain. A negative value of the velocity indicates that the fluid is driven on the electrodes in the direction of the traveling electric field. At low voltages, the fluid is driven in this direction, as expected by the low voltage theory of ac electroosmosis. ${ }^{14,15}$ We refer to this mode of behavior as normal pumping. At higher voltages, the fluid is driven in the opposite

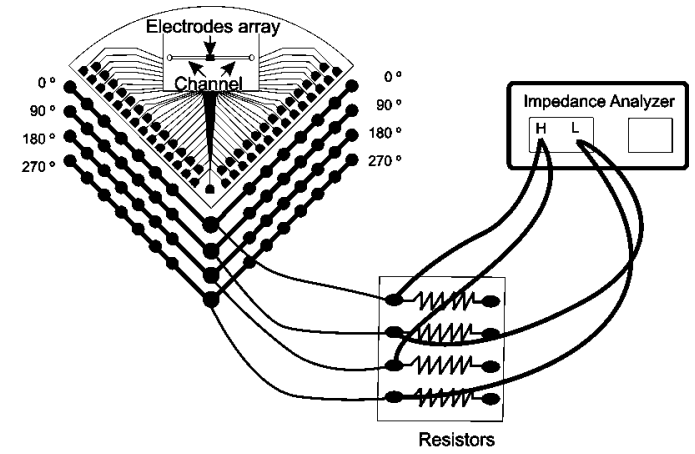

(b) Connections for the impedance measurement. Electrodes corresponding to phases $0^{\circ}$ and $180^{\circ}$ are connected to one wire and those corresponding to $90^{\circ}$ and $270^{\circ}$ to another

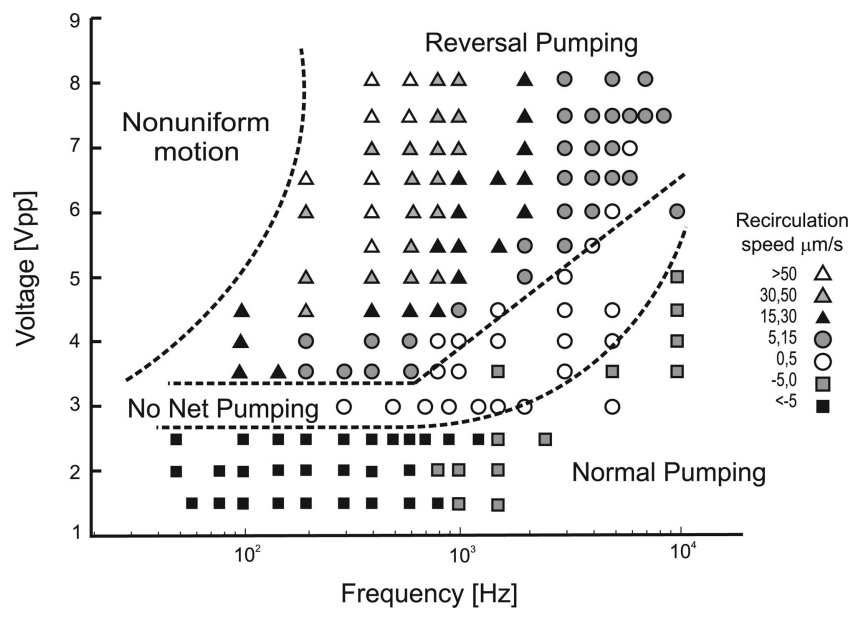

Figure 5. Velocity map for the traveling-wave array of $20 \mu \mathrm{m} \mathrm{Pt}$ microelectrodes in plane voltage versus frequency. Velocities are measured at a height of $120 \mu \mathrm{m}$ above the electrodes (Figure 3). Four different fluid flow behavior regions can be distinguished.

direction, a mode of behavior we designate as reverse pumping. The physical mechanism responsible for this behavior is not clear at present.

The Figure shows four distinguishable regions corresponding to normal pumping, reverse pumping, no net pumping, and nonuniform motion. There is a region of voltage where the pumping mechanism changes from normal to reverse pumping. A distinct net flow was not clearly observed in this region, although strong fluid rolls occurred over the electrodes. We designate this region as no net pumping. At low frequencies and for voltages where the reverse pumping occurs, there is a region where nonuniform fluid motion is observed. The fluid pattern is fully $3-\mathrm{D}$, and the profile of Figure 3 is not observed. This region is designated nonuniform motion. Electrolytic bubble generation was observed inside this region for sufficiently high voltage and low frequency. A similar flow map was observed for the 20$\mu \mathrm{m}$-wide titanium electrode array. ${ }^{21}$ However, we note that there are some qualitative differences because for the titanium array (1) electrolysis occurred at low frequencies before the fluid flow became nonuniform; (2) typical voltages for flow reversal were higher; and (3) there was a characteristic frequency of maximum velocity in the reverse pumping mode.

Figure 6 shows the fluid velocity as a function of frequency for array A at different voltages. The fluid flow behavior strongly 


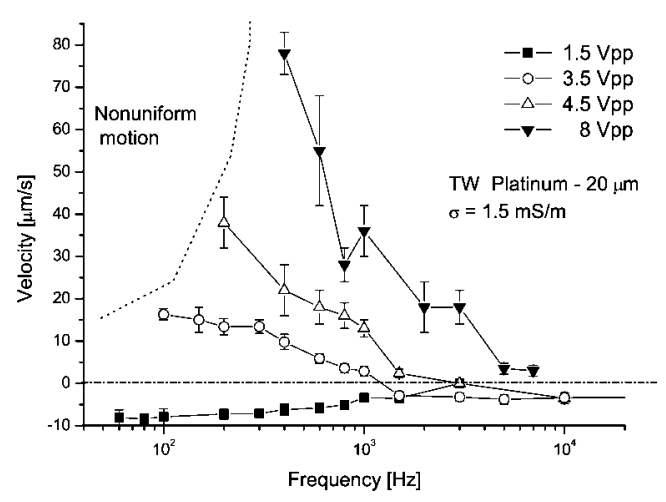

Figure 6. Velocity versus frequency for the $20 \mu \mathrm{m}$ array.

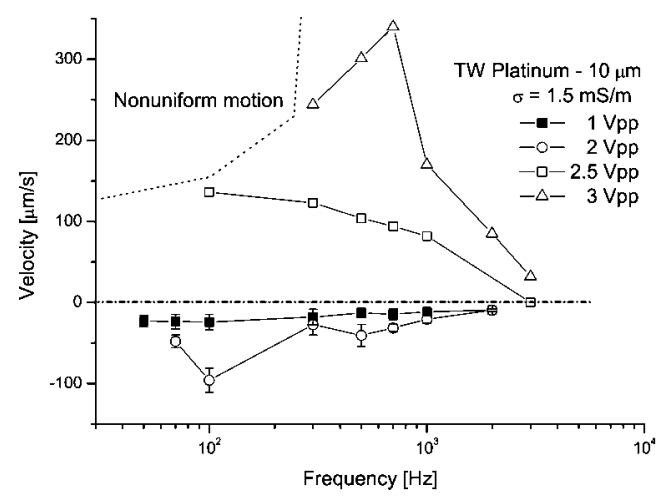

Figure 7. Velocity versus frequency for the $10 \mu \mathrm{m}$ array.

depends on frequency. In the reverse pumping region, we do not see any local velocity maximum. As the frequency decreases, the velocity increases until we reach the region of nonuniform motion. In the region of normal motion, a maximum absolute velocity seems to be present around $80 \mathrm{~Hz}$. However, the size of the error bars makes identification of the maximum rather difficult. In previous experiments using arrays of titanium electrodes, ${ }^{15,21}$ a velocity maximum was clearly observed. We propose two reasons for the different behavior: (a) Titanium electrodes are known to possess an oxide layer. The effect of this layer is modeled as an extra capacitance in series with the diffuse layer capacitance, which therefore shifts the typical frequency for ACEO to high values. (b) Platinum electrodes are far from the ideal perfectly polarizable electrodes, the converse of titanium electrodes. Electrochemical reactions easily occur when platinum electrodes are used. The typical frequency for ACEO is expected to be lower when Faradaic reactions are present. ${ }^{20,23}$

The same measurements were carried out with traveling-wave array B (10- $\mu \mathrm{m}$-wide electrodes). The qualitative behavior is similar to that of array A. Reverse pumping is observed at voltages at and above $2.5 \mathrm{~V}_{\mathrm{pp}}$. Nonuniform flow is observed in the reversepumping region when the frequency is low. Figure 7 shows the fluid velocity as a function of frequency for array B at several voltages. A quantitative difference between arrays A and B is that the velocities observed for array B were much greater than those observed for array A at the same voltages. This indicates that the velocity is greater for smaller electrodes. In the normalpumping region, a maximum in velocity appears at a frequency of around $100 \mathrm{~Hz}$. This is different for reverse pumping where a maximum is found at higher frequencies. Errors bars are not plotted; errors are large at velocities larger than $100 \mu \mathrm{m} / \mathrm{s}$ and can obscure the maximum in velocity. Furtheremore, because

(23) Olesen, L. Ph.D. Thesis, Technical University of Denmark, 2006.
Table 1. Threshold Voltages for Reverse Pumping for $f \leq 1 \mathrm{kHz}$

\begin{tabular}{cccc}
\hline & Ti $20 \mu \mathrm{m}$ & Pt $20 \mu \mathrm{m}$ & Pt $10 \mu \mathrm{m}$ \\
\hline threshold voltage & $4 \mathrm{~V}_{\mathrm{pp}}$ & $3.5 \mathrm{~V}_{\mathrm{pp}}$ & $2.5 \mathrm{~V}_{\mathrm{pp}}$
\end{tabular}

the apparent maximum is close to the nonuniform motion region, the maximum might not be significant because the flow is not uniform at these frequencies.

The reproducibility of the velocity measurements is influenced by effects such as aging of the electrodes; see ref 18 . Nevertheless, the threshold voltage for the appearance of flow reversal is very reproducible. The threshold voltage was observed to be more or less independent of frequency below $1 \mathrm{kHz}$ and increased with frequency above. The threshold voltage, deemed to be the lowest value at which flow reversal is clearly observed, is shown in Table 1 for three cases: the titanium $20-\mu \mathrm{m}$-wide electrode array (previously investigated in ref 21) and the two platinum electrode arrays studied here. The values shown in Table 1 are for frequencies below $1 \mathrm{kHz}$.

\section{Electrical Measurements}

4.1. Electrical Impedance. Measurements of the impedance of the electrode-electrolyte system were carried out by connecting the electrodes corresponding to phases of 0 and $180^{\circ}$ to one wire and those corresponding to 90 and $270^{\circ}$ to another (Figure 4b). A frequency sweep is performed for every measurement. The excitation voltage is kept low enough $\left(100 \mathrm{mV}_{\text {rms }}\right)$ that the liquid properties are not altered. Figure $8 \mathrm{a}, \mathrm{b}$ shows the impedance modulus and phase for array $\mathrm{B}$ before the traveling-wave potential is applied (full lines). This impedance represents a reference state for the electrical response of the electrode-electrolyte system. Each time the liquid was renewed, these impedance values were reproduced. The impedance was also measured after the array was used with specific traveling-wave signals for 5 min: the impedance at low frequencies $(<1 \mathrm{kHz})$ was found to be much smaller than that of the reference state whereas at high frequencies $(>100 \mathrm{kHz})$ it was only slightly smaller. Dotted lines in Figure 8a,b show the impedance modulus and phase measured immediately after removing the traveling wave potential $\left(3 \mathrm{~V}_{\mathrm{pp}}\right.$ and $100 \mathrm{~Hz}$ ) after it had been applied for $5 \mathrm{~min}$. Note that the time required for a frequency sweep is much shorter than the typical time of variation of the impedance (tens of seconds). This means that the quasi-equilibrium electrical properties of the system can be measured with time. It was observed that the fluid stopped immediately after the TW signal was switched off -the fluorescent particles did not move. Therefore, fluid flow does not occur during the impedance measurements; therefore, the impedance changes cannot be attributed to movement of the fluid.

There is the possibility that a temperature increase due to Joule heating could affect the impedance measurements. The total heat dissipation per unit time is given by the volume integral of $\sigma E^{2}$. At steady state, the heat flux is the value of this integral per unit surface area $k|\nabla T| \approx \sigma E^{2} l$, where $k$ is the electrolyte thermal conductivity and $l$ is the typical length of the electric field, which is on the order of the electrode width. If the gradient of temperature is written as $\nabla T \approx \Delta T / H$, with $H$ being the height of the channel, then an estimation of the temperature increment is given by $\Delta T \approx \sigma V^{2} H / k l$ with $E \approx V / l$. For the parameters in the experiments $(\sigma=1.5 \mathrm{mS} / \mathrm{m}, H=200 \mu \mathrm{m}, l=10 \mu \mathrm{m}, k=$ $0.56 \mathrm{~W} / \mathrm{m} \mathrm{K}$ ) and a typical voltage amplitude of $2 \mathrm{~V}$, we estimate $\Delta T \approx 0.2 \mathrm{~K}$. This small change in temperature cannot account for the very significant changes in impedance that were observed.

Figure 9 shows the impedance modulus and phase at discrete frequencies $(100 \mathrm{~Hz}, 800 \mathrm{~Hz}, 17.6 \mathrm{kHz}, 129.2 \mathrm{kHz}, 1 \mathrm{MHz})$ 


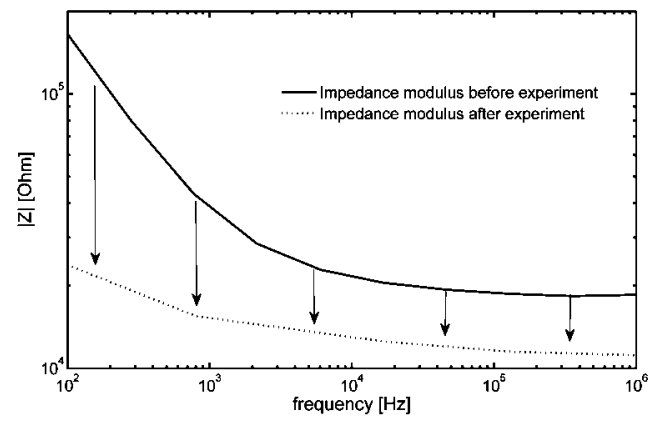

(a) Impedance modulus versus frequency. The measurements after the TW signal show a strong decrease at low frequencies.

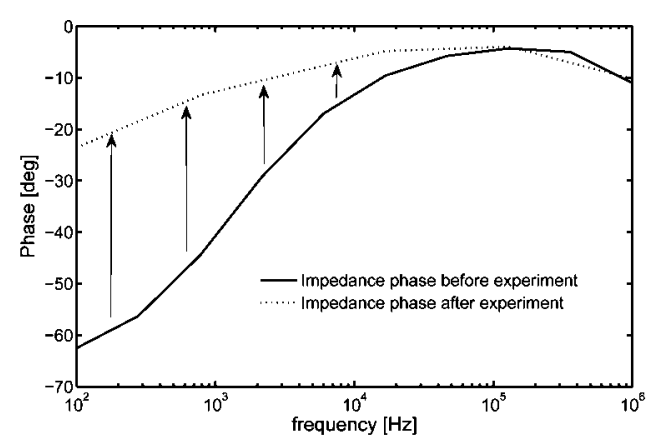

(b) Impedance phase versus frequency. The measurements after the TW signal show that the ratio of in-phase to out-of-phase currents increased at low frequencies.

Figure 8. Impedance measurements I: The impedance was measured before any signal was applied ( - ) and immediately after the cessation of a TW signal of $3 \mathrm{~V}_{\mathrm{pp}}$ and $100 \mathrm{~Hz}$ that had been applied for $5 \mathrm{~min}(\cdots)$.

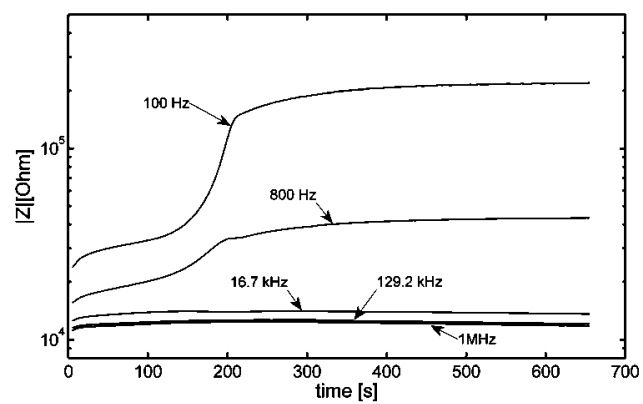

(a) Impedance modulus versus time. The impedance recovers the reference value after $\sim 200$ s. All electrodes connected (55)

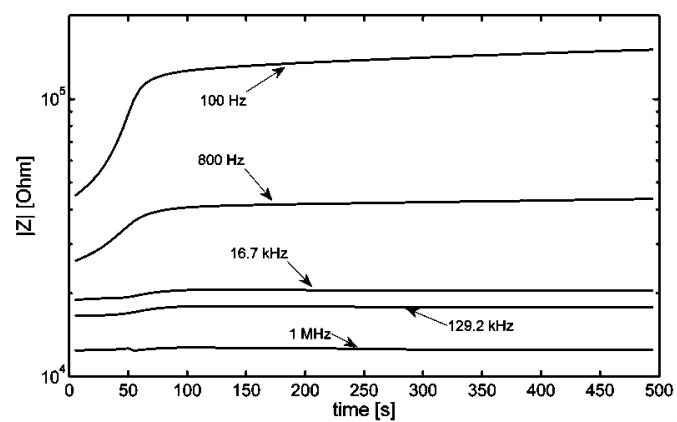

(c) Impedance modulus versus time. The impedance recovers the reference value after $\sim 50 \mathrm{~s}$. Half of the electrodes connected (27)

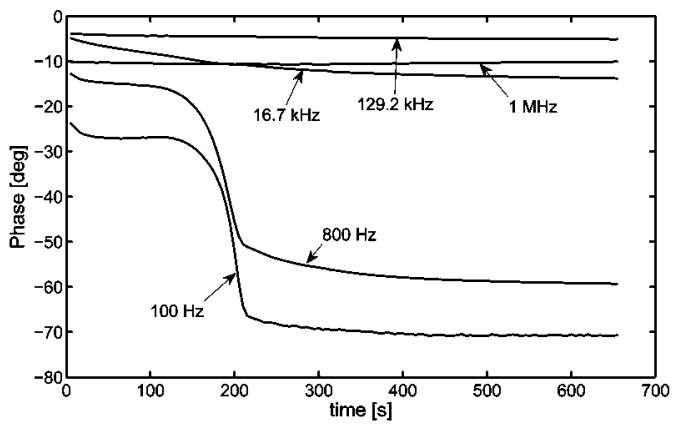

(b) Impedance phase versus time. The impedance recovers the reference value after $\sim 200 \mathrm{~s}$. All electrodes connected (55)

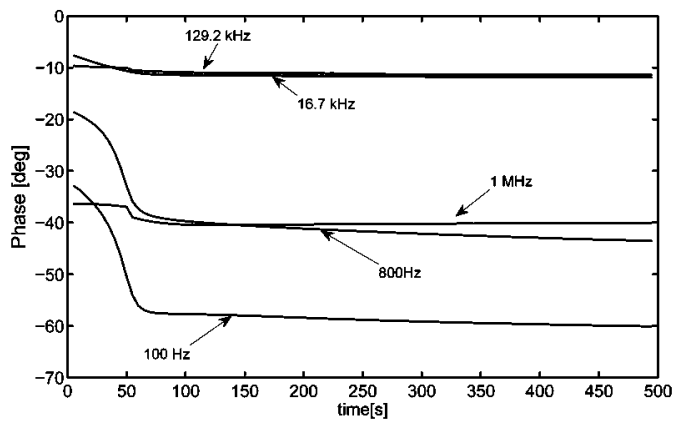

(d) Impedance phase versus time.The impedance recovers the reference value after $\sim 50 \mathrm{~s}$. Half of the electrodes connected (27)

Figure 9. Impedance measurements II: Impedance at several frequencies versus time for two different array lengths. The impedance was measured after the cessation of a TW signal of $3 \mathrm{~V}_{\mathrm{pp}}$ and $100 \mathrm{~Hz}$ that had been applied for 5 min.

versus time for array $\mathrm{B}$ after a TW signal $\left(3 \mathrm{~V}_{\mathrm{pp}}\right.$ and $\left.100 \mathrm{~Hz}\right)$ was applied for 5 min. Figure 9a,b shows measurements where all electrodes (55) were connected, and Figure 9c,d shows measurements where only half of the electrodes are connected (27). Note that, according to Figure 7, nonuniform fluid motion is to be expected with this signal. It is observed that the decrease in the impedance at $100 \mathrm{~Hz}$ is very large and quickly increases during the first $200 \mathrm{~s}$ and then slowly approaches the reference values (i.e., the impedance value before the TW signal was applied). At $800 \mathrm{~Hz}$, the initial impedance decrement is also very clear whereas at higher frequencies the decrease is not as strong (on a log scale), although it is present. The impedance phase changes are also very clear. The phase decrease is large at low frequencies, indicating that the ratio of in-phase to out-of-phase currents increases. These impedance observations mean that changes in the liquid properties are occurring. They also indicate that these changes are greatest near the electrodes. In effect, the impedance at low frequencies depends mainly on the properties of the solid-liquid interface and at higher frequencies the bulk properties of the liquid dominate the impedance. For very high frequencies $(>1 \mathrm{MHz})$, the properties of the entire system (including wires, connections, glass, etc.) govern the impedance.

Figure 9c,d shows impedance versus time when only half of the electrodes are connected (27 electrodes vs 55 ) so that the 


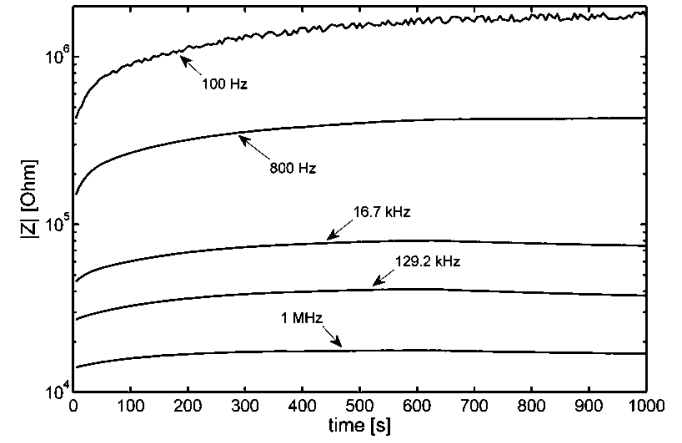

(a) Impedance modulus versus time after $3 \mathrm{~V}_{p p} 1 \mathrm{kHz}$

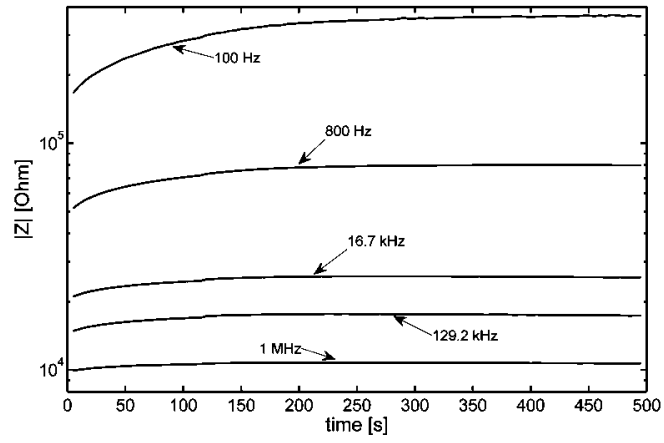

(b) Impedance modulus versus time after $1.5 \mathrm{~V}_{p p} 100$ $\mathrm{Hz}$

Figure 10. Impedance measurements III: Impedance at several frequencies versus time. The impedance was measured after cessation of two different TW signals: (a) $3 \mathrm{~V}_{\mathrm{pp}}, 1 \mathrm{kHz}$ applied for $5 \mathrm{minh}$ and (b) $1.5 \mathrm{~V}_{\mathrm{pp}}, 100 \mathrm{~Hz}$ applied for $5 \mathrm{~min}$.

array in this experiment is half the length of the previous measurements. There again appears to be a strong decrease in the impedance at low frequencies, but the recovery to the reference value is faster. Because the time for recovery of the impedance is given by the time for the species to diffuse to produce a homogeneous liquid, a shorter array should recover more rapidly. The microfluidic channel is much longer than the electrode array (Figure 4). This means that on both sides of the array there is a "reservoir" of fresh liquid. After switching off the signal, we expect to have complete homogenization when there is enough time for the liquid from the sides to reach the middle. Therefore, a faster recovery is expected for the shorter array. The typical time for a given chemical species to diffuse a length $L$ is $t \approx$ $L^{2} / D$, where $D$ is the diffusion coefficient of the species. The rapid increment of the impedance that was previously observed (within $200 \mathrm{~s}$ ) is now observed during the first $50 \mathrm{~s}$. This typical time for recovery is roughly 4 times shorter when the array is half the length. The diffusion coefficient can be estimated as $D$ $\approx(1 \mathrm{~mm})^{2} /(200 \mathrm{~s})=5 \times 10^{-9} \mathrm{~m}^{2} / \mathrm{s}$, which is typical of ionic species in water, supporting this assumption.

Figure 10a shows the impedance modulus versus time after a signal of $3 \mathrm{~V}_{\mathrm{pp}}$ and $1 \mathrm{kHz}$ (reverse-pumping region) was applied for $5 \mathrm{~min}$. The decrease in impedance is also very clear at low frequencies but is not as strong as found for $3 \mathrm{~V}_{\mathrm{pp}}$ and $100 \mathrm{~Hz}$, the region corresponding to nonuniform motion.

Figure 10b shows the impedance versus time after a signal of $1.5 \mathrm{~V}_{\mathrm{pp}}$ and $100 \mathrm{~Hz}$ was applied for $5 \mathrm{~min}$. The array is operating in the normal-pumping region, and changes in the impedance are still appreciable despite the fact that the voltage is substantially lower than the threshold voltage for this array. It could be inferred that changes in impedance are observed only for voltages at or above the threshold. This is not the case, and Figure 10b shows that the impedance also changes at signals corresponding to the normal-pumping mode. In general, the changes in impedance increase with increasing voltage and/or decreasing frequency. The decrement in the impedance at frequencies greater than 10 $\mathrm{kHz}$ indicates that the bulk conductivity is increasing. This increase means either an increase in ionic strength or an exchange of high-mobility species for lower-mobility species or a combination of the two (most likely). We expect the conductivity increase to be stronger near the electrodes because electrochemical reactions occur there. The strong decrement of the impedance modulus and the change in the impedance phase for lower frequencies seem to indicate that either the charge-transfer resistance has strongly decreased or the surface capacitance has increased. These changes can be driven by an increase in ionic strength and/or an increment in reactive high-mobility species such as $\mathrm{H}^{+}$and $\mathrm{OH}^{-}$.

From the net change in liquid properties, we infer that electrochemical reactions at the electrodes are not symmetrical with time. The rectifying properties of electrode-electrolyte interfaces is a well-known phenomenon ${ }^{24}$ and could be related to this observation. In ref 25 , current rectification was measured above a certain threshold current density - the threshold current increased with frequency. The estimated current densities in our experiments are of the same order of magnitude as these threshold current densities.

4.2. Current Measurements: Transient Measurements. The electrical current was measured when the traveling wave signal was applied to array A as indicated in the Experimental Details section (Figure 4b). The actual current was measured when the TW signal was applied, a completely different measurement to that described in the previous section. Linear behavior in the current-voltage characteristics was found at low voltage (below $1.5 \mathrm{~V}_{\mathrm{pp}}$ ). At higher voltages, nonlinearities appear, and the current increase with voltage was faster than for the linear case. This is observed as a decrease in the apparent impedance. Other researchers working with high sinusoidal currents have observed that the apparent impedance decreases as the current amplitude increases. ${ }^{25,26}$ More remarkably, we measured a time-dependent electrical current amplitude (i.e., the current was oscillating at the driving frequency $I(t)=I_{0} \cos (\omega t)$ with an amplitude $I_{0}$ that changed slowly with time). The characteristic time for this slow variation was on the order of minutes, which is very much greater than the period of the ac signal. In array B, we observed the current amplitude changing with time at and above $2 \mathrm{~V}_{\mathrm{pp}}$. In Figure 9, the current amplitudes are shown at several voltages versus time for array A at a frequency of $100 \mathrm{~Hz}$. Figure 11a shows similar data at $1 \mathrm{kHz}$. These measurements were performed as follows: starting from $0.5 \mathrm{~V}_{\mathrm{pp}}$, the current amplitude versus time is measured with a lock-in amplifier. The lock-in samples data until the current reaches a stationary value, then the signal is switched off and new liquid is flushed through the channel. The voltage is increased to a stationary value. Then, the signal is switched off, and new liquid is flushed through the channel. The voltage is increased in $0.5 \mathrm{~V}_{\mathrm{pp}}$ increments, the signal is switched back on, and the lock-in measures the current amplitude until a new stationary value is reached. Currents at $100 \mathrm{~Hz}$ show

(24) O'dea, J. J.; Osteryoung, R. A.; Osteryoung, J. G. Anal. Chem. 1994, 66, 798-801.

(25) Geddes, L. A.; Foster, K. S.; Reilly, J.; Voorhees, W. D.; Bourland, J. D.; Ragheb, T.; Fearnot, N. E. IEEE Trans. Biomed. Eng. 1984, 9, 669-672.

(26) Schwan, H. P. Ann. Biomed. Eng. 1992, 20, 269-288. 


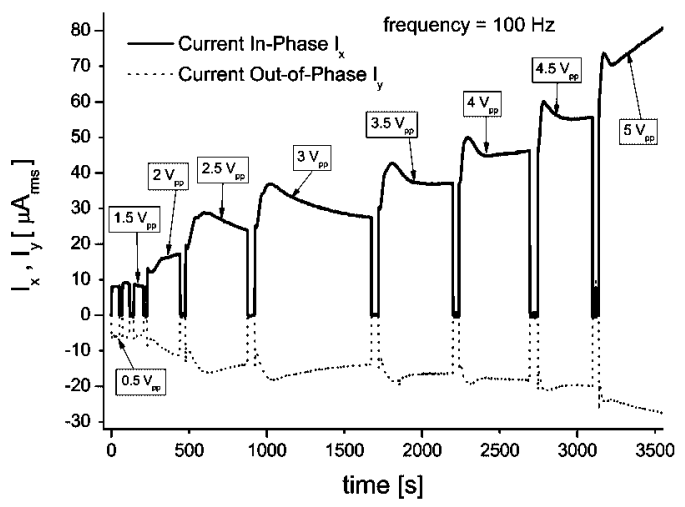

(a) Electrical current vs time at $100 \mathrm{~Hz}$

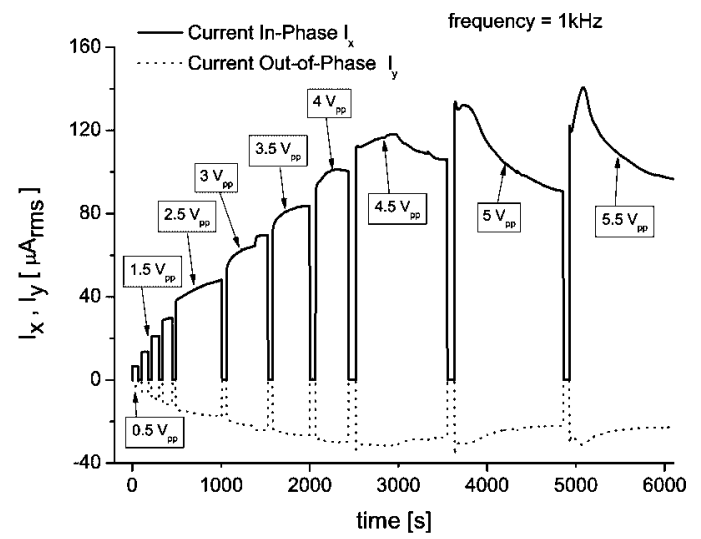

(b) Electrical current vs time at $1 \mathrm{kHz}$

Figure 11. Electrical current versus time during the application of TW signals of different amplitudes.

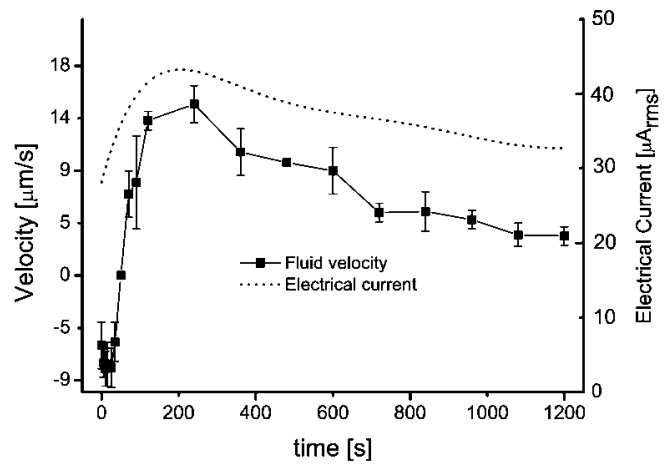

(a) Velocity and electrical current vs time at $3 V_{p p}$ and $400 \mathrm{~Hz}$

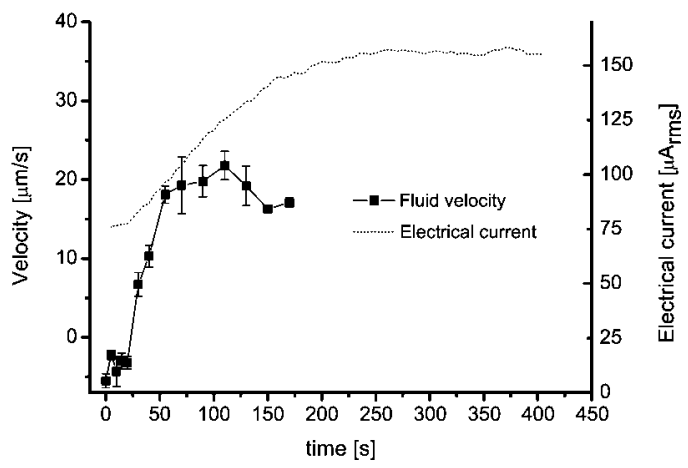

(b) Velocity and electrical current vs time at $3 V_{p p}$ and $700 \mathrm{~Hz}$

Figure 12. Electrical current and fluid velocity versus time during the application of a TW signal.

peaks that are typical of Faradaic currents. At $1 \mathrm{kHz}$, these peaks were not clearly observed. Note that the capacitive current increases with frequency so the total current is expected to be greater at $1 \mathrm{kHz}$ and any Faradaic currents could be masked by capacitive currents.

Observations of the flow behavior for signals corresponding to the no-net-pumping region of Figure 5 are shown in the next two Figures. For array A at a voltage of $3 \mathrm{~V}_{\mathrm{pp}}$ and at $400 \mathrm{~Hz}$, we observed that the fluid started to move slowly in the normalpumping mode and, after around $2 \mathrm{~min}$, the direction was reversed (Figure 12a). Measurements of the current amplitude are also plotted, and both velocity and current are strongly correlated. Figure $12 \mathrm{~b}$ shows the same transient behavior at $3 \mathrm{~V}_{\mathrm{pp}}$ and 700 $\mathrm{Hz}$. At this frequency, the current amplitude is roughly double that at $400 \mathrm{~Hz}$, and Faradaic currents are likely to be masked by capacitive currents. The inversion of the net fluid flow was reproducible if new liquid was flushed through the channels. If after applying any of these signals the liquid was not changed, then the fluid started to move in the reverse-pumping mode, indicating some hysteresis. Notice that this reversal of flow in time was not observed for signals outside the no-net-pumping region.

\section{Discussion}

The experimental work performed with traveling wave arrays with electrodes of different metals and sizes seems to confirm that the fluid flow map in ref 21 represents the general behavior of these systems. Velocity data were obtained from videos recorded during the $20 \mathrm{~s}$ after the channel was filled with new liquid and the signal was switched on. We used this protocol to obtain the flow map in Figure 5. The low-voltage measurements are not expected to depend on this protocol, but at higher voltages this may not be true. In particular, the region of no net pumping is most likely to be affected. As shown in Figure 12, for signals of amplitude $3 \mathrm{~V}_{\mathrm{pp}}$, the fluid moves first in the normal direction and after 50-100 s it moves in the reverse direction. The flow map of Figure 5 is interpreted as the early behavior of the micropump, and it is a useful description of the phenomena, although not complete because transient behavior is also present.

Experimental observations with DI water as the working fluid were also carried out, and reverse pumping was found at threshold voltages slightly higher than those for $\mathrm{KCl}$ solutions with $\sigma=$ $1.5 \mathrm{mS} / \mathrm{m}$. Under all experimental circumstances studied, the fluid velocity reverses if the voltage is sufficiently high and the frequency is optimal. However, new qualitative observations are found when platinum electrodes are used. At low frequencies, close to the region of reverse pumping, nonuniform fluid flow is observed. The flow patterns indicate that electrohydrodynamic (EHD) instabilities are probably taking place. Gradients in the conductivity of the electrolyte can give rise to such instabilities. ${ }^{27-29}$ EHD instabilities in microchannels with ac driving signals have recently been studied. ${ }^{30}$ These authors found a strong frequency dependence when the base state of the fluid electrical

(27) Baygents, J. C.; Baldessari, F. Phys. Fluids 1998, 10, 301-311.

(28) Chen, C. H.; Lin, H.; Lele, S. K.; Santiago, J. G. J. Fluid Mech. 2005, $524,263-303$.

(29) Lin, H.; Storey, B. D.; Oddy, M. H.; Chen, C.-H.; Santiago, J. G. Phys. Fluids 2004, 16, 1922-1935.

(30) Boy, D. A.; Storey, B. D. Phys. Rev. E 2007, 76, 026304. 
conductivity is considered to be steady. A steady conductivity gradient can appear by thermal or electrochemical effects, as could happen in the present case. Other instabilities such as those studied by Rubinstein and co-workers ${ }^{31}$ may appear at high current densities. They are associated with the depletion of electrolyte near the interfaces, which seems to be opposite to the present case. The impedance measurements at low frequencies show a strong decrease, which is particularly strong after applying signals corresponding to the nonuniform motion region. This supports the assumption that EHD instabilities can occur because, as stated above, the liquid properties are mainly changing near the electrodes and gradients in conductivity (i.e., ion concentrations) should appear. Nevertheless, changes in the electrolyte impedance are clear after applying the traveling potential even for signals corresponding to the normal pumping region. These changes in liquid properties are not taken into account in the current theories of ac electroosmosis. They may be important in the explanation of the fluid flow observations. Gradients in conductivity under an applied field can yield net charge; consequently, they are a source of bulk forces in the liquid. Experiments indicate that the relative importance of these bulk forces compared to forces in the diffuse layer increases because of Faradaic currents. In effect, our measurements show that double-layer impedance decreases with voltage amplitude, which means that the ratio of the voltage drop across the double layer to the total applied voltage is smaller.

In ref 32, the authors demonstrate ac electroosmotic fluid flow and observed a reversal of the movement as the voltage increases. They attributed the flow reversal to a Faradaic charging mechanism in which Faradaic currents would generate net charge on the liquid side of the interface with the same sign as the electrode charge. A net electrical force on the fluid would appear that is opposite to that predicted by ACEO theory. This view of Faradaic charging is also present in recent work on dc-biased ac electroosmosis. ${ }^{33} \mathrm{We}$ argue that this mechanism contradicts the assumption of the electroneutrality of electrolytes at frequencies below the charge relaxation frequency because counterions are not considered in this view. Gauss' law shows that the liquid bulk is quasi-electroneutral on the micrometer length scale $\rho / e$ $\ll \Sigma_{i} c_{i}$. In effect, the relative difference in ion number densities for a $1: 1$ electrolyte is given by $\left(c_{+}-c_{-}\right) /\left(c_{+}+c_{-}\right)=\nabla \cdot \varepsilon \mathbf{E} /$ $e\left(c_{+}+c_{-}\right)$and is very small for saline solutions on the micrometer length scale for typical applied electric fields. ${ }^{34,35}$

Crowding of ions due to their finite size $\mathrm{s}^{36,37}$ and Faradaic currents through the interface might have the effect of limiting the voltage drop across the EDL. However, forces in the bulk due to the induced charge should become more important as the applied voltage increases. In refs 38-40, forces beyond the Debye length were also considered for the generation of fluid flow near the electrodes. From the continuity equation for the electrical current $(\nabla \cdot J=0)$ and the quasi-electroneutrality assumption, the free charge density $\rho$ in the bulk is given by ${ }^{41}$

$$
\frac{\rho}{\varepsilon}=\frac{\nabla \sigma \cdot \nabla \phi}{\sigma}+\frac{\sum_{i} z_{i} e D_{i} \nabla^{2} c_{i}}{\sigma}
$$

where $\sigma$ is the liquid conductivity, $\phi$ is the electric potential, $e$ is the proton charge and $z_{i}, D_{i}$, and $c_{i}$ are the ionic valence,

(31) Rubinstein, I.; Zaltzman, B. Phys. Rev. E 2000, 62, 2238-2251.

(32) Lastochkin, D.; Zhou, R.; Wang, P.; Ben, Y.; Chang, H.-C. J. Appl. Phys. 2004, 96, 1730-1733.

(33) Wu, J. IEEE Trans. Nanotechnol. 2006, 5, 84-89.

(34) Saville, D. A. Ann. Revi. Fluids Mech. 1997, 29, 27-64.

(35) Castellanos, A.; Ramos, A.; González, A.; Green, N. G.; Morgan, H. J. Phys. D: Appl. Phys. 2003, 36, 2584-2597.

(36) Kilic, M. S.; Bazant, M. Z.; Ajdari, A. Phys. Rev. E 2007, 75, 021502. (37) Kilic, M. S.; Bazant, M. Z.; Ajdari, A. Phys. Rev. E 2007, 75, 021503. diffusion coefficient, and concentration of species $i$, respectively. The charge in eq 1 could give rise to a nonzero time-averaged force $\langle\rho E\rangle \neq 0$. It should be noted that this time average is performed over the signal period, which is much smaller than the typical time of variation of the impedance (tens of seconds). In at least the following two cases, we expect $\langle\rho E\rangle \neq 0$ :

(a) The term $\nabla^{2} c_{i}$ has a time-varying component with the same frequency of the electric field. In this case, the time average is not zero because it is the product of two sinusoidal functions of the same frequency. It could produce net free charge in a layer of thickness $\delta=\sqrt{ } D / \omega$, with $\omega$ being the angular frequency of the applied field. For typical values of $D \approx 10^{-9} \mathrm{~m}^{2} / \mathrm{s}$ and $\omega \approx$ $10^{3} \mathrm{rad} / \mathrm{s}$, this distance is on the order of micrometers $\delta \approx 1 \mu \mathrm{m}$. The force due to this term has been considered in refs 42 and 43 for particle motion on top of ac-polarized electrodes. This second term in eq 1 is zero for a binary symmetrical electrolyte $\left(\left|z_{+} D_{+}\right|=\left|z_{-} D_{-}\right|\right)$.

(b) The first term in eq 1 could give rise to a nonzero timeaveraged force if the time-averaged conductivity gradient is nonzero $\langle\nabla \sigma\rangle \neq 0$, as would seem to be indicated by the impedance measurements. If the liquid conductivity is higher near the electrodes than in the bulk, then the situation is similar to that encountered in ac electrothermal flow. ${ }^{44,45}$ The vertical conductivity gradient leads to fluid flow in the direction of the reverse pumping mode; however, the characteristic frequency of this motion is on the order of the relaxation frequency $(f=\sigma / 2 \pi \varepsilon)$. At higher voltages and frequencies, conductivity gradients can be generated electrochemically and may be responsible for certain observations and behavior. Nevertheless, at the frequencies of the present observations $(f \ll \sigma / 2 \pi \varepsilon)$, the influence of this mechanism is not clear. According to this mechanism and for $f \ll \sigma / 2 \pi \varepsilon$, the fluid velocity would increase with frequency, contrary to observations, although we should take into account that the rate of electrochemical reactions decreases with increasing frequency. For a given vertical conductivity gradient, the fluid velocity can be estimated from the expression $v \approx\left(\varepsilon V_{0}^{2} \omega \tau / 8 \eta\right)(\partial$ $\ln \sigma / \partial z),{ }^{44}$ where $\tau=\varepsilon / \sigma$. This is around $100 \mu \mathrm{m} / \mathrm{s}$ for a conductivity gradient of $\partial \ln \sigma / \partial z=0.1 / \mu \mathrm{m}$ and an applied signal of $4 \mathrm{~V}_{\mathrm{pp}}$ at $1 \mathrm{kHz}$. Longitudinal gradients of conductivity were not considered in this treatment, but they could actually be relevant in the generation of fluid flow. The importance of the nature of the electrochemical reactions at the electrodes and their effect on the fluid flow is worth noting. Reactions that generate ionic species or, more precisely, increase the conductivity lead to flows in the opposite direction to those reactions that reduce the conductivity (consumption of ions).

One question is what possible electrochemical mechanism could cause the observed changes in conductivity. The applied voltages are close to those that produce water electrolysis, which is used in all of the experiments, and this could change the local $\mathrm{pH}$ and conductivity of the electrolyte. ${ }^{38,39,46} \mathrm{We}$ are currently working on other ways to explore changes in $\mathrm{pH}$. Preliminary observations using a $\mathrm{pH}$-sensitive dye show that the solution

(38) Trau, M.; Saville, D. A.; Aksay, I. A. Langmuir 1997, 13, 6375-6381. (39) Ristenpart, W. D.; Aksay, I. A.; Saville, D. A. Langmuir 2007, 23, 40714080 .

(40) Ristenpart, W. D.; Aksay, I. A.; Saville, D. A. J. Fluid Mech. 2007, 575, 83-109.

(41) Newman, J. S.; Thomas-Alyea, K. E. Electrochemical Systems; WileyInterscience: Hoboken, NJ, 2004.

(42) Sides, P. J. Langmuir 2001, 17, 5791-5800.

(43) Fagan, J. A.; Sides, P. J.; Prieve, D. C. Langmuir 2004, 20, 4823-4834.

(44) Melcher, J. R.; Firebaugh, M. S. Phys. Fluids 1967, 10, 1178-1185.

(45) Fuhr, G.; Hagedorn, R.; Müller, T.; Benecke, W.; Wagner, B. J. Electromech. Syst. 1992, 1, 141-146.

(46) Fiedler, S.; Hagedorn, R.; Schnelle, T.; Richter, E.; Wagner, B.; Fuhr, G. Anal. Chem. 1995, 67, 820-828. 
becomes acidic, implying that there are reactions that lead to either $\mathrm{H}^{+}$production (most likely if the conductivity increases) or $\mathrm{OH}^{-}$consumption. As mentioned in section III in ref 21 , the velocity profile generated by a TW microelectrode array of titanium electrodes was studied and was found to correspond to Figure 3. The net fluid flow is generated at the level of the electrodes, but this does not mean that the electrical forces are confined inside the EDL. If bulk forces exist and act within a distance of typically the size of the electrodes, then a similar fluid flow profile would be obtained. Comparing the results for titanium and platinum electrodes indicates that the role of the metal seems important: the threshold voltage is higher for titanium electrodes, and a well-defined maximum velocity (with frequency) in reverse pumping mode is seen. Titanium is a metal that readily forms a highly resistant oxide layer, and this was accounted for in the linear ACEO calculations presented in refs 15 and 16. Because Faradaic reactions are likely to be responsible for the changes in the liquid properties, the chemical nature of the metal may be important. The thin oxide layer on titanium acts as a passivation layer, so higher voltages are required to generate Faradaic currents on titanium, consistent with the observed increase in threshold voltage.

\section{Conclusions}

The platinum microelectrode arrays with two different electrode width and spacings had similar flow characteristics in the voltage-frequency domain. Both showed four distinct regions: normal pumping, reverse pumping, no net pumping, and nonuniform motion. In both arrays, at low voltages the liquid flow was in accordance with predictions given by ac electroosmotic theory (normal pumping). Typical frequencies were lower for array A, the large electrodes. At voltages above a threshold, net fluid flow was reversed. Reversal flow occurred at lower voltages for the array with smaller electrodes (array B). There does not appear to be a local maximum velocity in the reverse pumping mode; the velocity increases with decreasing frequency until the nonuniform region is reached. Comparison with previously published data using titanium microelectrode arrays shows that the threshold voltage was greater for these electrodes.
Typical frequencies for normal pumping were lower for the platinum electrode array than for the titanium electrode array. A local velocity maximum in the reverse pumping region is found for the titanium array. One noticeable difference between the two metals is that nonuniform motion was not found for the titanium devices and bubble formation occurred before nonuniform motion was observed.

Impedance measurements show that liquid properties change after the application of the TW signals; these changes are greatest near the electrodes. As a result of this, gradients in conductivity appear, which would give rise to bulk forces in the liquid. If the conductivity is higher at the level of the electrodes, then forces in the direction of reverse pumping would be generated. An estimate of this velocity indicates that these forces can be important. The observation of nonuniform motion could be due to EHD instabilities caused by conductivity gradients.

Measurements of the electrical current during pumping showed transient behavior. At specific voltages, around the threshold voltage, simultaneous measurements of fluid velocity and electrical current showed a strong correlation. Faradaic currents are probably responsible for this transient behavior and for the gradients in conductivity. When DI water was used, the results were similar, indicating that electrochemical reactions of water could be responsible for the observations.

An analysis of the experimental results provides new insight into the underlying mechanisms of pumping electrolytes with microelectrode arrays. Future experimental work should focus on the electrochemical characterization of the system when energized with high ac voltages, together with an identification of which of the different forces, forces in the bulk or forces in the double layer, are responsible for the observations. From a theoretical point of view, it would be interesting to determine how the forces caused by gradients in conductivity can give rise to fluid motion in these electrode arrays.

Acknowledgment. We acknowledge the financial support of the Spanish government agency DGCyT and Junta de Andalucía under contracts FIS2006-03645 and FQM-241, respectively.

\section{LA $800423 \mathrm{~K}$}

